\title{
DIE KLEINE SCHEIDEGG
}

\author{
ERNST WINKLER
}

«Von Lauterbrunnen nach Grindelwald giebt es zwey sehr unterschiedene Wege. Niederwärts das Thal hinaus nach Zweylütschinen und von da durchs Lütschenthal geht fahrbar der eine; aufwärts über die Scheideck der Wengen oder Wengernalp geht der andere, doch nur Pferden und Fußgängern gebahnte. Zum erstenmal ward dieser im Jahr 1771 von Herrn Pfarrer Wyttenbach, in Begleit des berühmten Freundes von Johannes Müller, des Herrn von Bonstetten, zu Gunsten der Reisenden unserer Zeit erprobt, und dann auf das nachdrücklichste empfohlen. Kein Reisender sollte unterlassen ihn zu versuchen. Er ist gefahrlos. Er ist nicht über 8 Stunden weit. Er ist der höchste von unseren oberländischen Bergpässen. Er gewährt, wie kein anderer, einen erhabenen Anblick des herrlichsten Schneegebirges. Man schreitet eine Stunde lang steil empor bis zu dem zerstreuten Berghüttendorf Wengen. Alsdann führt müheloser der Pfad eine geraume Zeit längs einer breiten Abflachung des Berghangs über dem Lauterbrunnenthale hin nach dem Trümletenthal, welches fast rechtwinklicht von der Jungfrau nach jenem zur Tiefe dringt. Plötzlich aber wendet sich der Weg und zieht eine Stunde lang, mitten durch eine gastfreundliche Sennerey von zahlreichen Hütten, im steten Anschauen der Jungfrau und beyder Eiger, östlich auf den obersten Grat der Scheideck, und von hier windet er sich in den Boden des Grindelwaldthales, das unausgesetzt vor dem spähenden Blicke liegt, und jenseits die große Haslischeideck zur Gränze hat.»

Mit diesen Worten leitete der Professor für Philosophie an der Universität Bern, Dichter und Geograph Johannes Rudolf Wyß «der Jüngere»(1781-1830), nicht nur die wohl erste eingehendere und eindrückliche Schilderung eines der bekanntesten und am meisten begangenen schweizerischen $\mathrm{Paßübergänge} \mathrm{ein.} \mathrm{Sie} \mathrm{markieren} \mathrm{zugleich} \mathrm{den}$ Beginn seiner eigentlichen Kulturlandschaftsentwicklung, die vom selten benützten Jäger- und Hirtenpfad zur modernsten Hochgebirgsbahn- und Hotelregion führte. Im zitierten Text ist hiefür vor allem bezeichnend: Er ist gefahrlos. Dieser Satz war zweifellos nötig, weil frühere - in der Regel meist flüchtige - Würdigungen die «Wängenoder Lauterbrunner Scheideck» als mühselig und gefahrvoll bezeichnet hatten. Wy erschloß mit seiner beruhigenden Bemerkung die Route recht eigentlich dem Fremdenverkehr, wenn auch schon früher bekannte einheimische und fremde Besucher, so der von ihm als «Veteran unserer Alpenschilderer und Alpen-Naturforscher» gerühmte Jakob Samuel Wyttenbach, der dänische Dichter J. Baggesen, der Franzose M. T. Bourrit, die Engländer William und Thomas Coxe, Lord Byron und andere sie als «landschaftliche Schönheit» empfohlen hatten.

Inzwischen waren die Alpen als solche bereits zum $Z$ iel vieler Besucher geworden. Das touristische Zeitalter hatte begonnen. In diesem «Jahrhundert der Reiselust schwoll der Besucherstrom von Jahrzehnt zu Jahrzehnt an und wurde gegen dessen Ende zur Flut. $\mathrm{Zu} \mathrm{Fuß,} \mathrm{auf} \mathrm{dem} \mathrm{Sattelpferd,} \mathrm{auf} \mathrm{dem} \mathrm{Maultier} \mathrm{und} \mathrm{im} \mathrm{Tragstuhl,} \mathrm{begleitet} \mathrm{von}$ Führern und Trägern zogen erholungsuchende und schönheitsdurstende Seelen sonder Zahl über den $\mathrm{Pa}$. Die kostspieligste Beförderungsart war die im Tragstuhl. Es benötigte normalerweise vier Mann zum abwechslungsweisen Tragen einer Person über die Kleine Scheidegg. Der Fall kam aber auch vor, daß sich die doppelte Anzahl der stärksten Talleute weigerten, eine offenbar vollgewichtige fremde Frau zu ,tragstuhlen'. 
Am Rande dieser oft fröhlichen Karawanenzüge stellten sich, hier wie anderswo, auch die Zaungäste ein, Alphornbiäser, Liedersänger, Blumenverkäuferinnen, Echoböllerschützen und Hackbrettspieler, die die Brosamen nahmen, welche vom Tisch der Hablichkeit einer sorglosen Zeitepoche abfielen. Diese Menschenflut brachte der zum Teil armen Bevölkerung beider Täler willkommenen Verdienst. Die Lohnsätze für die Erwerbenden waren schon in der ersten Hälfte des Jahrhunderts behördlich geregelt. Ein Pferd oder ein Maultier nebst Knecht kostete von Lauterbrunnen über die Wengernalp nach Grindelwald per Tag 6 Schweizer Livre (alter Berner Franken, $1851=$ 1 Fr. 44 Rp.), ein Träger von Lauterbrunnen bis auf Wengernalp 4 Schweizer Livre.» (H. Michel)

Noch 1846 fand es indessen das «Geographisch-statistische Handlexikon des Schweizerlandes» von J. J. Leuthy, das der Großen Scheidegg einen rühmenden Artikel gewidmet hatte, nicht nötig, für die «Kleine» dasselbe zu tun. Es begnügte sich beim Stichwort Wängernalp mit dem lakonischen Hinweis: «Ein Reitweg führt darüber von einem Thal in das andere»; zehn Jahre später freilich schreibt es: «,der Alpberg' ist triftenreich, mit Hütten und Heuschobern besetzt, und wird wegen der Verbindung zwischen beiden Thälern und der herrlichen Aussicht, besonders auf die Jungfrau, jährlich von vielen Tausenden von Reisenden begangen. Ein ziemlich bequemer Reitweg führt von Grindelwald nach Lauterbrunnen. Auf der Lauterbrunner Seite steht etwa 50 Minuten unter der Scheideck (6284 F. ü. M.) das Hotel de la Jungfrau, 5330 F. ü. M., ein ländliches Wirtshaus, in welchem Reisende gewöhnlich den Sonnenaufgang abzuwarten pflegen, und wo Byron einen Theil seines 'Manfred' dichtete. Auch auf der Scheideck, wo sich der volle herrliche Blick auf die Jungfrau bietet, steht ein jetzt nicht mehr bewohntes Wirtshaus». Damit war angedeutet, daß der $\mathrm{Pa} \beta$ die erste Periode eines zukunftsreichen Tourismus erlebt und auch schon kritische Entwicklungsabschnitte hinter sich hatte.

Die zweite Periode setzte gegen Ende des 19. Jahrhunderts ein, indem damals die bahntechnische Erschließung des Jungfraugebietes begann. Mit diesem Ereignis wurde es nun auch möglich, die statistische Verifizierung der «Tausende von Reisenden»zu erbringen, von der die frühere Zeit gesprochen hatte. Nachdem 1890 die BerneroberlandBahnen - mit Ausgangspunkt in Interlaken/Ost und Endstation Lauterbrunnen und Grindelwald - den Betrieb aufgenommen hatten und nach der Eröffnung der Bergbahnen Lauterbrunnen-Mürren 1891 und -Schynige-Platte 1893 konnte schon im letztern Jahre auch die Wengernalp-Bahn von Lauterbrunnen nach Grindelwald über die Kleine Scheidegg die ersten Gäste befördern. Die Zahl der in diesem Jahr registrierten Passagiere betrug 37 742. Sie steigerte sich bis 1900 auf 88799 , und von da an kennzeichnet mit nur durch die Weltkriege und die Weltwirtschaftskrise um 1930 bedingten Unterbrüchen, ein steter Frequenzanstieg die Entwicklung der Bahn (1910: 140 067, 1915: 19670,1920 : 92 669, 1925: 286 168, 1929: 361 759, 1930: 333 474, 1932: 174 013, 1937: 465 879, 1940: 196 026, 1950: 522 626, 1960: 1179 748, 1963 : 1525717 ), deren Bedeutung damit deutlich markiert wird. Die Bahn fährt mit 18\% Steigung von Lauterbrunnen (796 m) (früher bestand eine ältere Linie mit $25 \%$ ) über Viadukte und durch Tunnels bergwärts. Nach dauernd wechselndem Tiefblick auf den berühmten Wasserfall des Staubbachs und auf das Lauterbrunnental erreicht sie den weltbekannten Sommer- und Winterkurort Wengen $(1274 \mathrm{~m})$, von wo die Fahrt über Weiden und durch Tannenwälder mit Blick auf Jungfrau (4158 m), Breithorn (3782 $\mathrm{m})$, Tschingelhorn $(3577 \mathrm{~m})$ und Gspaltenhorn $(3457 \mathrm{~m})$ nach Wengernalp $(1873 \mathrm{~m})$ weiterführt. Im Blick auf das imposante Dreigestirn Eiger (3970 m), Mönch (4099 m) und Jungfrau (4158 m) weiterfahrend, gelangt der Reisende schließlich bei $2061 \mathrm{~m}$ zur $\mathrm{Paßhöhe} \mathrm{der} \mathrm{Kleinen} \mathrm{Scheidegg} \mathrm{selbst,} \mathrm{wo} \mathrm{er} \mathrm{in} \mathrm{gut} \mathrm{geführten} \mathrm{Hotels} \mathrm{(Bellevue,} \mathrm{Des}$ Alpes) mit Restaurant sowie im Bahnhofbuffet Unterkunft findet. Wer nicht auf demselben Weg zurückkehren will, hat Gelegenheit, mit der gleichen Bahn an Alpenrosenfluren, wetterzerzausten Arven und durch Schutzgalerien, über Alpiglen (1615 m) an 


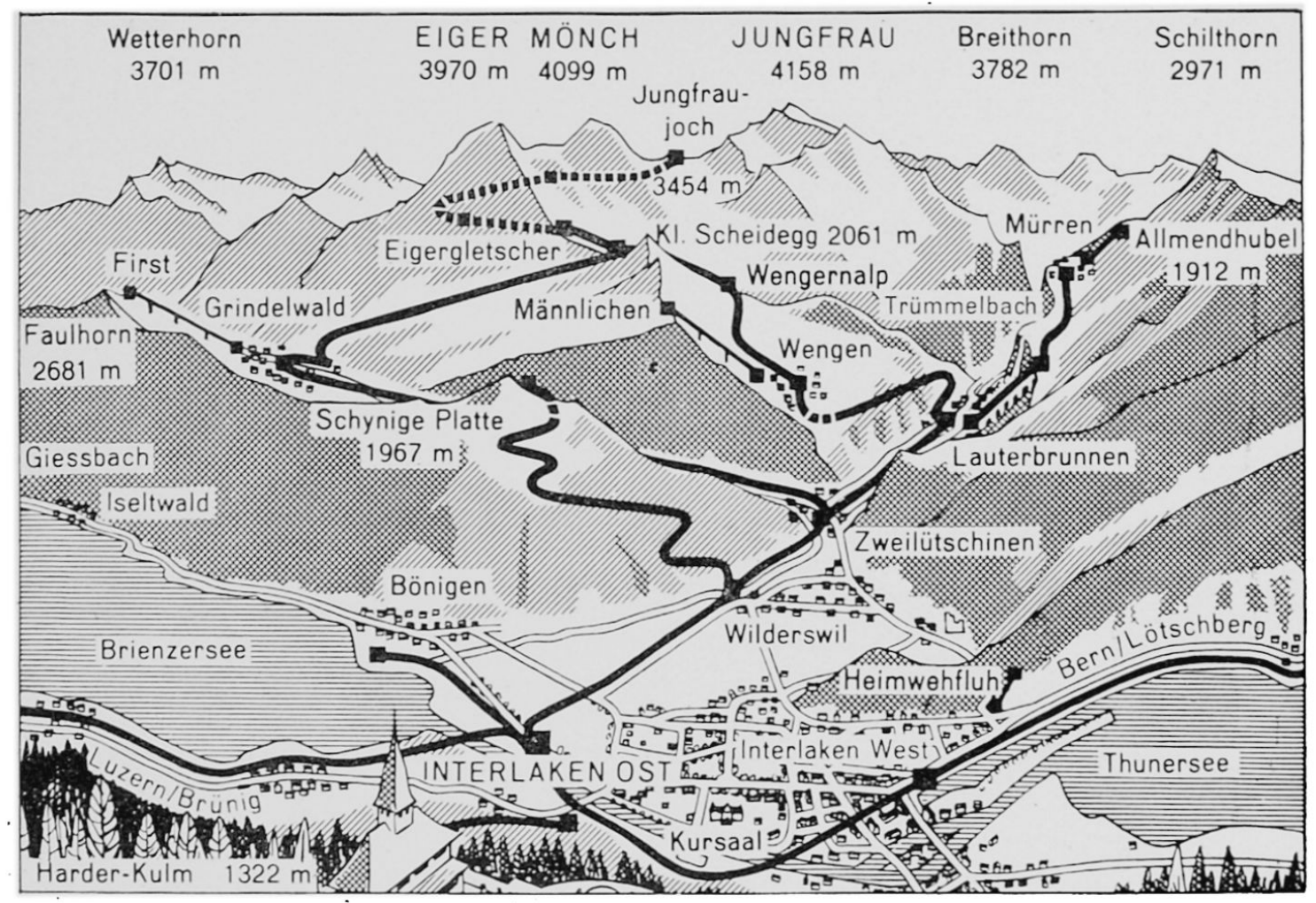

Schema-Panorama des Jungfraugebietes. In dessen "Kern» die Kleine Scheidegg als Ausgangspunkt anziehungsreichster Hochgebirgstouren der Schweiz und der Alpen.

Cliché: Direktion Wengernalpbahn, Interlaken

der schroffen Eigernordwand vorbei mit Blick auf das zerzackte Wetterhorn $(3701 \mathrm{~m})$ nach Grindelwald und zu Tale zu fahren, wobei Gefälle bis zu 25\% überwunden werden.

Es ist klar, daß die Wengernalpbahn das ehemalige Landschaftsbild der Gegend erheblich gewandelt hat, was von den im vorigen angedeuteten Tatsachen abgesehen daraus ersichtlich wird, daß auf der ganzen $19091 \mathrm{~m}$ langen Linie 7 Tunnels (mit insgesamt $1471 \mathrm{~m}$ Länge), 27 Brücken (insgesamt $489 \mathrm{~m}$ Weite), 77 Ausweichstellen, 14 Unterführungen, 1 Überführung und 117 Niveauübergänge sowie 11 Stationen und eine Waldschneise von gegen $1,5 \mathrm{~km}$ nötig wurden. Ferner erforderte nach $\mathrm{H}$. Michel die Strecke Lauterbrunnen-Scheidegg allein $114840 \mathrm{~m}^{3}$ Erdbewegung, $23341 \mathrm{~m}^{3}$ Felseinschnitte, $8608 \mathrm{~m}^{3}$ Stütz- und Futtermauern in Mörtel, $2675 \mathrm{~m}^{3}$ Sickerungen, $9415 \mathrm{~m}^{3}$ Brücken und Durchlässe sowie $11655 \mathrm{~m}^{3}$ Beschotterung. Das Paßgelände selbst, eine teils flache, teils reich gegliederte «Rundhöckerlandschaft», ein Glied der von der Sefinenfurgge (im Westen) bis zur Großen Scheidegg (im Nordosten) sich hinziehenden schiefrig-flyschigen Längssenke zwischen dem Nordrand des Aaremassivs und den Helvetischen Decken ( $H$. Gutersohn), erhielt naturgemäß die stärkste Umprägung insofern, als sich auf ihm neben der höchsten Bahnstation das Hotelgewerbe entfaltete. Nachdem dasselbe noch im 19. Jahrhundert, wie erwähnt, verschiedentlichen Gunstschwankungen unterlegen war, konnte das Geographische Lexikon zu Beginn des 20. melden, daß nunmehr auf der Kleinen Scheidegg zwei Gasthöfe und ein Restaurant bestünden, die alljährlich von «ungezählten Touristenscharen» besucht würden. 
Diese positive Entwicklung war zweifellos zu einem guten Teil dem Bau der Jungfraubahn zu verdanken. Deren Anfänge reichen gleichfalls zum Ende des 19. Jahrhunderts zurück, die Gesamteröffnung fällt jedoch auf das Jahr 1912. Sie wurde in den Jahren 1896-1912 nach Ideen des bekannten Fabrikanten A. Guyer-Zeller errichtet, wobei im Unterschied zur Wengernalpbahn der größte Teil der Betriebsstrecke $(7561 \mathrm{~m}$ von $9336 \mathrm{~m}$ ) in zwei Tunnel zu liegen kam, so daß die Physiognomie der Landschaft, ungeachtet der kühnen Anlage der Bahn im ganzen, entschieden weniger verändert wurde als diejenige der Gegend, durch welche die Züge jener fahren. Die Jungfraubahn als die Königin der Bergbahnen darf noch heute als die höchste Bahn Europas gelten, da sie mit ihrer Endstation Jungfraujoch 3454 m erreicht. Auch ihre Frequenzen verzeichnen seit Eröffnung 1898 einen nur durch die Krisenzeiten unterbrochenen Anstieg von 1966 auf 378202 (1962) beförderte Reisende, woraus hervorgeht, daß durchschnittlich ein Fünftel bis ein Drittel der von der Wengernalpbahn beförderten Passagiere auch die Jungfraubahn benützten.

Mit der Vollendung der Jungfraubahn war eine dritte Entwicklungsperiode der Landschaft der Scheidegg eingeleitet, die in gewissem Sinne durch die Eröffnung des Ganzjahresbetriebes im Jahre 1925, das heißt durch die Verankerung des Wintersportes in der Region, gekrönt wurde. Mit den inzwischen in Hotels umgewandelten zwei Gasthöfen, die etwa 120 Betten führten, trat sie in die Reihe der weltbekannten Wintersportplätze ein; sie gingen um diese Zeit in den Besitz der Familie von Allmen über, die sie noch heute unter dem Namen «Scheidegghotels» erfolgreich führt. In der letzten Zeit vergrößerte sie die Bettenzahl auf 150 und führte überdies ein Touristenlager ein, das ebenfalls gut frequentiert ist, zumal außer den Bahnreisenden noch jährlich zahlreiche Fußgänger aus beiden Talgründen (Grindelwald, Lauterbrunnen) den Paßübergang der Kleinen Scheidegg erwandern. Die Scheidegg-Hotels (Hotel Bellevue und Des Alpes) verzeichneten folgende Ankünfte: 1934: 3322, 1940: 1069, 1950: 5256, 1960 : 4868, 1962: 5643 und 1963: 5309 (wozu in letzterem Jahre noch 2455 Reisende des Touristenlagers gezählt wurden). Ihnen entsprachen 1934: 21 921, 1950: 14 130, 1960: 25023 und 196324788 Logiernächte (plus 2731 im Touristenlager), so daß auch die Hotels eine den Bahnen parallele Frequenzentwicklung erlebten.

Die Bahnen als Wegbereiterinnen des Fremdenverkehrs im Jungfraugebiet und damit als stärkste Faktoren der Landschaftsumformung des Scheidegg-Gebietes erfuhren in den rund 60 bis 70 Jahren ihres Bestehens selbst nicht geringe Wandlungen. Sie waren einerseits durch die Entwicklung der Technik, anderseits auch durch die gesteigerten Anforderungen und den Funktionswandel des Tourismus bedingt. War die Wengernalpbahn am 20. Juni 1893 als reine Zahnradbahn (System Riggenbach) mit Dampflokomotiven dem Betrieb übergeben worden, so wurde sie 1910 elektrifiziert und durch eine sicherere Anfahrt Lauterbrunnen-Wengen verbessert. In den Jahren 19481954 erfolgte dann die Verwirklichung eines weitern Erneuerungsprogrammes, das die Beschaffung von 7 Schnelltriebwagen, die Erstellung einer Spitzkehre auf der Kleinen Scheidegg zur Gestaltung eines flüssigeren Verkehrs Wengen-Grindelwald und die Inbetriebnahme einer zweiten Schneeschleudermaschine umfaßte. Auf der Kleinen Scheidegg wurde zudem das Bahnhofbuffet umgebaut, wozu in Lauterbrunnen und Grindelwald neue Wagenremisen kamen. Außerdem wurden auf der Grindelwald rampe etappenweise Lawinenschutzbauten errichtet. Seit 1948 verkehren auf der Bahnlinie 18 neue, mit den letzten technischen Errungenschaften ausgerüstete Motorwagen; die Bahn verfügt ferner über 46 Personenwagen mit 2874 Sitzplätzen, über 19 offene und 9 geschlossene Güterwagen, 1 Langholzwagen, 3 Rollwagen, 12 Skitransportwagen, 2 Schneeschleudermaschinen und 3 Schneepflüge. Mit den technischen Neuerungen konnte die Fahrt Lauterbrunnen-Kleine Scheidegg auf 40, diejenige von Grindelwald nach der Kleinen Scheidegg auf 35 Minuten limitiert werden, was einer Fahrzeitverkürzung von 27 bzw. 23 Minuten entspricht. Die Geschwindigkeit beträgt für die Bergfahrt $17-22 \mathrm{~km} / \mathrm{h}$, für die Talfahrt $12-15 \mathrm{~km} / \mathrm{h}$. 


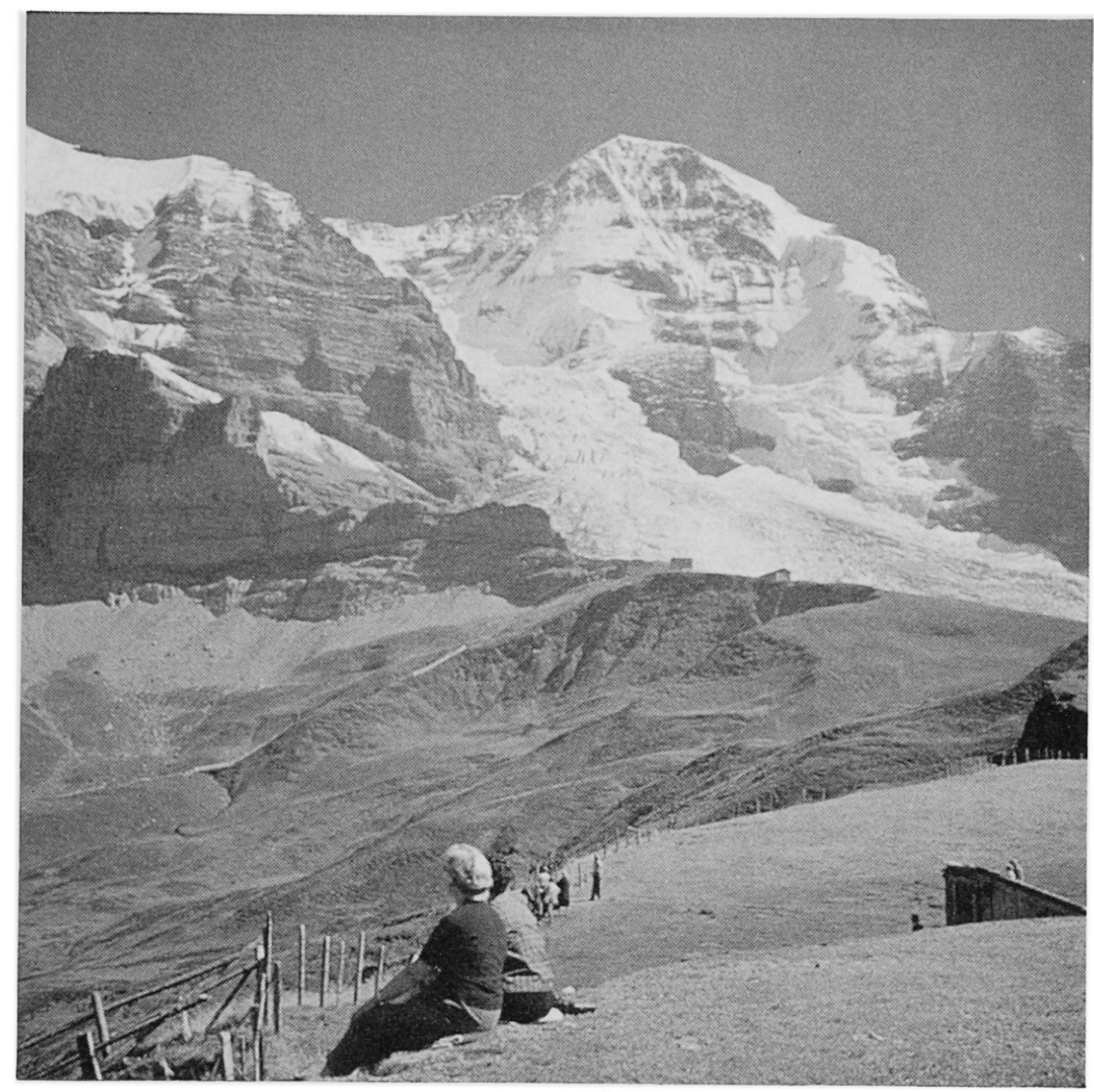

Photo: O. Beyeler, Bern

Seit den Anfängen des Fremdenverkehrs ist die Kleine Scheidegg der am meisten besuchte Paßübergang im Berner Oberland. In unmittelbarer Nähe erhebt sich der Mönch mit dem gewaltigem Strom des Eigergletschers zu seinen Füßen. 


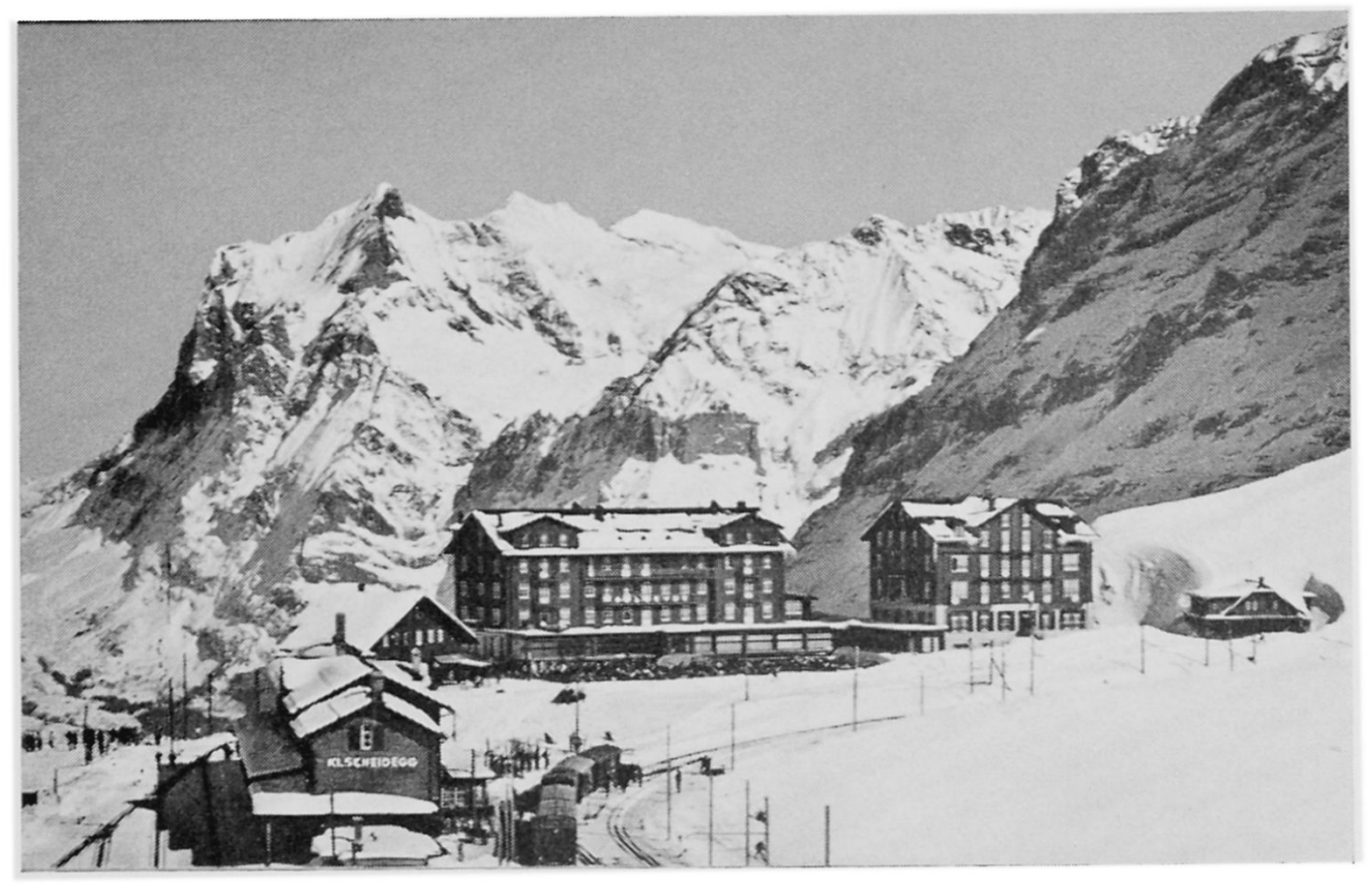

Die Kleine Scheidegg im Winter. Vorne die Station der Wengernalpbahn, daneben die Scheidegg Hotels, in der Mitte « Bellevue , rechts « Des Alpes». Im Hintergrund das Wetterhorn.

Photo S. Wyss-Konzett, Kleine Scheidegg

Auch die Jungfraubahn führte in den Jahren nach dem Zweiten Weltkrieg ein technisches Erneuerungsprogramm durch, da das früher gemischte Betriebssystem (Strecke Eismeer-Jungfraujoch Adhäsion, übrige Strecken Zahnrad) unzulänglich war. 1951 beseitigte man die Adhäsionsstrecke durch Einlegen von Zahnstangen und erstellte unterhalb der Station Eigergletscher Lawinengalerien. Ferner kamen 1955-1964 10 Schnelltriebwagen sowie 10 Personenwagen neuester Konstruktion zum Einsatz. Das Rollmaterial der Jungfraubahn besteht somit aus 6 elektrischen Lokomotiven, 10 Triebwagen, 22 Personenwagen mit 1560 Sitzplätzen, 8 Güterwagen, wovon 2 Wassertankwagen, 2 Skisportwagen, 1 Schneepflug und 1 Schneeschleuder. Die Tatsache, daß sie zu einem bedeutenden Forschungszentrum (Jungfraujoch) mit einem internationalen Forschungsinstitut (1931) und einem meteorologischen Observatorium (1937) führt, in welchen neben physiologischen, medizinischen, geologischen und meteorologischen namentlich auch geophysikalische Forschungen betrieben werden - an welchen sich Wissenschafter aus fast allen Ländern der Erde beteiligen - hat ihr in den letzten Jahren vermehrte Bedeutung verliehen, die übrigens noch in Steigerung begriffen ist.

Bei aller durchgreifenden Technifizierung der Hochgebirgslandschaft der Kleinen Scheidegg soll nicht vergessen werden, daß sie noch immer weitgehend Natur- und patriarchalische Wirtschaftsregion geblieben ist, trotzdem vom $\mathrm{Paßsattel}$ aus nach beinahe allen Richtungen zu Fuß oder per Bahn alljährlich Tausende von Menschen die umrahmenden Hochgebirgsgipfel Jungfrau, Mönch und Eiger, das Lauberhorn, den Männlichen und andere bevölkern. Noch immer zeichnen sich von den Gründen der Lütschinentäler her die durch die starke Vertikalgliederung der Erdrinde und ihre Folgen in Klima, Hydrographie und auch Böden hervorgerufenen Höhengürtel der 
«natürlichen»Vegetation: vom Buchen-, Buchen-Tannen-, Fichten- und Lärchen-Arven-Wald mit ihren Sonderformen etwa des Fichtenwaldes mit Heidelbeer-, Erikaoder Hochstaudenunterwuchs, über den Zwergstrauch-Tundrengürtel (die oberhalb Wengen und Grindelwald Alpweiden unterbrechen) bis zu den kahlen Schutt-, Firnund Schneegebieten der nivalen Zone deutlich ab, wiewohl sie Land- und Forstwirtschaft teilweise grundlegend verändert haben. Auch Land- und Forstwirtschaft selbst bestimmen noch weithin das Gelände; in der Wengernalp, die von Westen her bis zur Wasserscheide der Kleinen Scheidegg reicht und zugleich die politische, die Gemeindegrenze gegen Grindelwald markiert, kommt dies ebenso zum Ausd ruck wie auf der jenseitigen Abdachung im Alpgebiet von Wärgistal, das noch die einzigen Arvenbestände von Bedeutung im Landesteil trägt - wo vermutlich 1792 der letzte Bär der Gegend erlegt wurde.

So.verbinden sich im Gebiet der Kleinen Scheidegg und ihren Tälern eine großartige Natur, angestammte agrare Wirtschaftsweise und modernster Fremdenverkehr zusammen mit fortschrittlichster Wissenschaft zu einem landschaftlichen Gefüge, das zweifellos positive Weiterentwicklung in sich schließt und auch verdient. Den Ingenieuren und Unternehmern, welche das bedeutende Werk der Bahnen geschaffen haben, kommt hierbei ein nicht geringes Verdienst zu. Ihnen ist wesentlich zu verdanken, wenn Tausende von Menschen eines einmalig schönen Hochgebirgserlebnisses teilhaftig werden können, das sie sonst nur aus der Ferne kennengelernt hätten. Die Kleine Scheidegg freilich als $\mathrm{Pa}$, Ausgangspunkt und Schlußpunkt und damit letzten Endes unmittelbarste Quelle dieses Erlebnisses ist nicht Menschenwerk, sie ist ein Element der Natur, die dem Menschen immer die «Hand führt» (E. Egli) und der deshalb vor allen andern Dingen die «Palme» gebührt.

\section{QUELLEN}

H. Gutersohn: Geographie der Schweiz. Alpen II.Teil. Bern 1964. — Lüdi, W.: Die Pflanzengesellschaften des Lauterbrunnentales und ihre Sukzession. Zürich 1921. - Michel, H.: 50 Jahre Wengernalp-Bahn 1893-1943. Interlaken 1943. - Derselbe: Buch der Talschaft Lauterbrunnen. Interlaken 1950. - Wyss, J. R.: Reise in das Berner Oberland, zweite Abteilung. Bern 1817. - Die Bahnen im Jungfraugebiet. Interlaken 1964. - Verschiedene wertvolle namentlich statistische Auskünfte verdankt der Verfasser der Direktion der Bahnen im Jungfraugebiet, Herrn Direktor F. von Allmen-Seiler, Scheidegg Hotels sowie dem Eidg. Statistischen Amt in Bern.

\section{LA PETITE SCHEIDEGG}

Lorsque le col entre Lauterbrunnen et Grindelwald, entre le massif de la Jungfrau et du Männlichen, a seulement une importance secondaire dans le plan régional, il a gagné sous le nom de «Petite Scheidegg» (2061 m) une renommée internationale. La cause principale de sa plus grande fréquentation, est le magnifique panorama sur les sommets du groupe de la Jungfrau et sur des massifs enneigés voisins qui se présente au passage de celui-ci.

Les constructions des chemins de fer de Wengen (1891-1893) et de la Jungfrau (1893-1912) modifiaient sensiblement le paysage de la Scheidegg. Elle est devenue une station touristique internationale avec des hôtels sur place et dans sa banlieue. L'importance augmenta encore dès le début des sports d'hiver, en 1920 environ. Dès lors, la Petite Scheidegg est connue comme zone mondiale de tourisme dans laquelle séjournent plus d'un million de visiteurs par année. Dans la suite, le nombre des voyageurs transportés par le chemin de fer de Wengen entre 1893 et 1963 augmentait de 37742 à 1529717 ; le chemin de fer de la Jungfrau transportait en 1898 (ouverture de la première étape) 1966, en 1912 (l'achèvement de la ligne) 77626 et en 1963 (record) 274266 passagers. Les hôtels de la Scheidegg enregistraient en 193421291 , en 196324788 , respectivement 27519 logements (y compris les dortoirs), tandis que le nombre des arrivées pendant la même période augmentait de 3322 à 7764. Ces augmentations étaient interrompues par les deux guerres mondiales et par la crise économique 1930. Ces chiffres prouvent le développement continuel et progressif. 